\title{
Coronavirus Disease 2019 Infection among Children: Pathogenesis, Treatment, and Outcome
}

\author{
Krishna Rao Gurugubelli ${ }^{1}$ Ballambattu Vishnu Bhat ${ }^{2}$ \\ ${ }^{1}$ Department of Biochemistry, All India Institute of Medical Sciences, \\ Mangalagiri, Andhra Pradesh, India \\ 2 Department of Pediatrics and Neonatology, Aarupadai Veedu \\ Medical College and Hospital, Kirumambakkam, Puducherry, India \\ Address for correspondence Ballambattu Vishnu Bhat, MD, \\ Department of Pediatrics and Neonatology, Aarupadai Veedu Medical \\ College and Hospital, Kirumambakkam, Puducherry 607403, India \\ (e-mail: drvishnubhat@yahoo.com).
}

J Pediatr Intensive Care 2021;10:167-173.

\begin{abstract}
Keywords

- COVID-19 infection

- clinical features

- diagnosis

- treatment

- children

- neonates

Coronavirus disease 2019 (COVID-19) is a contagious disease that may lead to respiratory distress syndrome and even death. Neonates and children are most vulnerable population to COVID-19 infection; however, the infection is usually milder and has a better prognosis in pediatric patients compared with adults. It remains unclear why pediatric population is less symptomatic than adults. Children frequently experience respiratory infections and their immune system is in developing stage. However, large proportion of the asymptomatic pediatric population may contribute to transmission. This review explored several aspects of COVID-19 infection such as its epidemiology, its molecular pathogenesis with respect to angiotensin-converting enzyme 2 receptor and inflammatory mediators, intrauterine vertical transmission, imaging findings, and complications like cytokine release syndrome (multisystem inflammatory syndrome in children). We also looked at prognostic factors and treatment modalities like corticosteroids, RNA replicate inhibitors, protease inhibitors, Bruton tyrosine kinase inhibitor, that is, acalabrutinib and convalescent plasma therapy. Since there is no strong evidence for the intrauterine transmission, early isolation should be performed to protect a neonate from a COVID-19 infected mother. Development of vaccine and an effective antiviral drug are the need of the hour.
\end{abstract}

\section{Introduction}

Coronavirus disease 2019 (COVID-19) is a life-threatening disease causing massive public health problem worldwide. It has originated from Wuhan, China and is spreading in both developing and developed countries. The epidemic impact depends on the number of infected individuals, mode of transmission, and spectrum of clinical severity. ${ }^{1}$ The target organ for COVID-19 is believed to be the lungs; however, patients often have evidence of multiorgan dysfunction such as affecting heart, liver, kidney, stomach, brain, blood, and immune system. ${ }^{2,3}$ The COVID-19 infection causes clusters of severe respiratory illness similar to severe acute respiratory syndrome coronavirus (SARS-CoV). It is often associated with

received

June 22, 2020

accepted

September 2, 2020

published online

October 5, 2020 intensive care unit admission and high mortality. Patients usually develop upper respiratory tract signs and symptoms initially. ${ }^{3}$ About $2 \%$ of the people infected with COVID-19 are healthy carriers and this virus plays a key role in 5 to $10 \%$ of acute respiratory infections. Phylogenetic analysis revealed that "A and B" lineage of $\beta-\mathrm{CoV}$ ( $\mathrm{HCoVs}$ family) can cause common cold, respiratory tract infections, and extra respiratory infections with increased clinical severity, respectively (-Fig. 1). ${ }^{4}$

\section{Epidemiology}

Epidemiological classification of COVID-19 includes those at high, moderate, and low risk. High risk consists of close contact with confirmed COVID-19 cases within 14 days after onset.

(c) 2020. Thieme. All rights reserved. Georg Thieme Verlag KG, Rüdigerstraße 14,

70469 Stuttgart, Germany
DOI https://doi.org/ 10.1055/s-0040-1718417. ISSN $2146-4618$. 


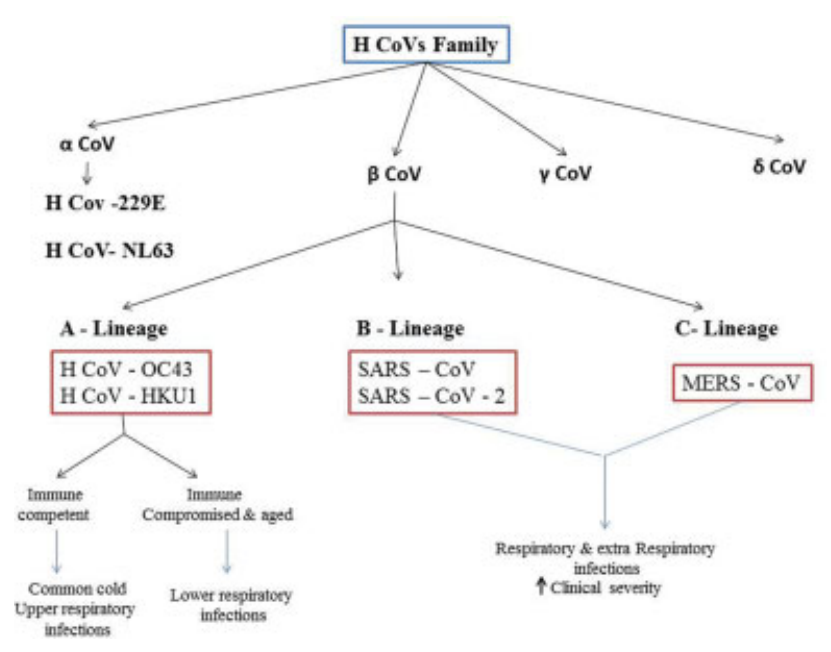

Fig. 1 Phylogenetic tree of human coronavirus. Human coronavirus classifies into four genera of coronavirus: $\alpha, \beta, \delta$, and $y$ coronavirus. The "A" lineage of $\beta$-CoV cause common cold, upper, and lower respiratory infections and the " $B$ " lineage cause respiratory and extra respiratory infections with high clinical severity. COVID-19 belongs to the " $B$ " lineage of $\beta-C o V$. COVID, coronavirus disease.

Medium risk consists of COVID-19 outbreak clusters in residential areas and community. Low risk consists of people in endemic areas outside the source of epidemic. ${ }^{5}$ COVID-19 clinical manifestations in children may be less severe compared with adults. This may be related to both host factors and exposure. Usually, children who are well cared for at home might have relatively less chances to expose themselves to pathogens and patients who are sick. Children frequently experience respiratory infections and may have higher level of antibody against viruses than adults. The immune system of children is in developing stage and it responds to pathogens differently compared with adult immune system. ${ }^{6}$ Some studies reported that boys are more vulnerable to COVID-19 compared with girls. ${ }^{7,8}$ But Dong et al did not observe significant difference between two genders. ${ }^{6}$ Common circulating HCoVs are seen in 4 to $6 \%$ of children hospitalized from acute respiratory tract infections. Children with age less than 3 years and those with heart disease are most often symptomatic. In later life, reinfections are more common. Compared with other respiratory tract infections, there is no decrease in $\mathrm{HCoV}$ s with increasing age. ${ }^{9}$ Children with COVID-19 may be mostly asymptomatic or only have gastrointestinal symptoms, pharyngitis, or cough. ${ }^{6}$

\section{Pathogenesis}

Structural analysis during SARS-CoV outbreak in 2002 has revealed specific molecular level interactions between spike protein receptor binding domain of SARS-CoV and its receptor angiotensin-converting enzyme 2 (ACE-2) in the host. Based on the available evidence on SARS-CoV and recent severe acute respiratory syndrome coronavirus 2 (SARS-CoV-2) sequence, Wan et al suggested that COVID-19 uses ACE-2 as its receptor. While binding certain critical residues in COVID-19 receptor binding motif(Gln493), it brings out positive interactions with human ACE-2 leading to infectivity. The phylogenetic analysis indicated the origin of 2019-nCoV from a bat and recognizes ACE-2 from a wide variety of animal species suggesting that all these species as potential intermediate hosts for novel coronavirus infections. ${ }^{10}$ ACE-2 receptors are expressed on epithelial cells of nasal cavity, type 2 alveolar cells of lungs, heart, kidney, and gastrointestinal tract. Lungs are more vulnerable to COVID-19 because of their large surface area. Patients with chronic obstructive pulmonary disease (COPD) are known to have increased expression of ACE-2 receptors leading to severe lung disease. ${ }^{11} \mathrm{Li}$ and Zhang reported that ACE-2 expression is not significantly different between healthy individuals and cases with COPD and asthma. But cases with underlying lung conditions possibly are at greater risk for extreme chronic airway disease or death. COVID-19 infected lung tissue expresses high concentration of ACE-2 receptors leading to chronic inflammatory response and pyroptosis (-Fig. 2). ${ }^{12}$

\section{Vertical Transmission}

Pregnancy involves a lot of physiological changes, which include compromised functional residual volumes, elevated diaphragm, altered cell immunity, and immunosuppressive state that make them more vulnerable to respiratory infections. Researchers tested breast milk, cord blood, amniotic fluid, and neonatal throat swab samples to detect the chance of intrauterine infection. All those samples were negative for COVID-19, which reflects that no vertical transmission occurrence of COVID-19 during pregnancy. ${ }^{13-15}$ The sequences of 2019-nCov-2 are almost identical and share $79.6 \%$ sequence identity to SARSCoV. ${ }^{16}$ During pregnancy, the fetus is protected from COVID-19 infection by dominant T-helper 2 (Th2) cells, which provide defense to the fetus and leave the mother susceptible to viral infections. ${ }^{13}$ Chen et al reported that COVID-19 has severe impact on neonates resulting in complications such as respiratory distress, premature labor, thrombocytopenia, fetal distress, distorted liver function, and sometimes death. ${ }^{14}$ Wang et al

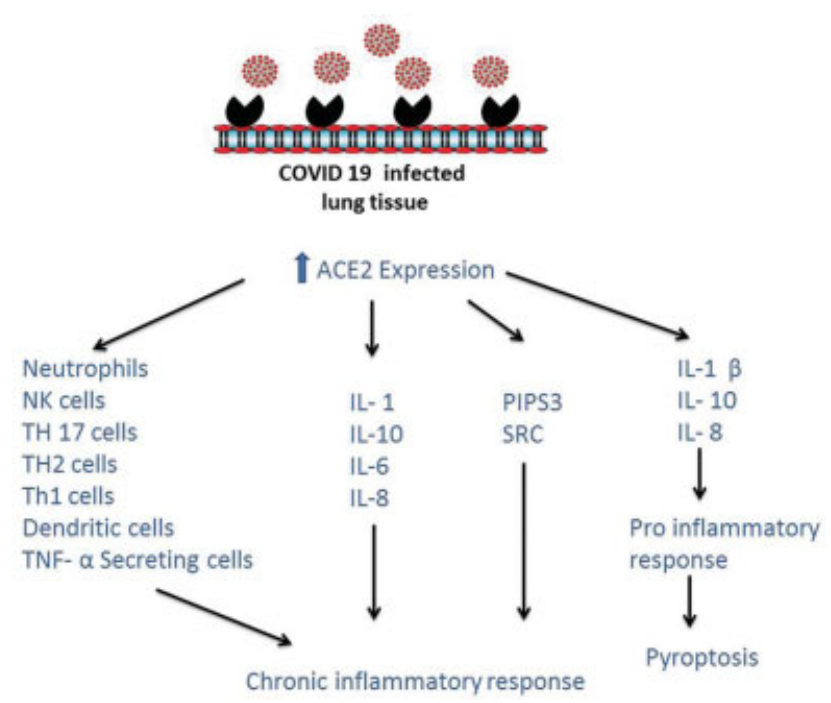

Fig. 2 Chronic inflammatory response and pyroptosis in COVID-19 infected lungs: increased ACE-2 expression leads to chronic inflammatory response and pyroptosis. ACE, angiotensin-converting enzyme; COVID, coronavirus disease. 
reported the first neonate born with SARS-nCo-2 infection in china, whose mother was COVID-19 positive. Both mother and newborn were detected with mild symptoms, although the placenta and cord blood samples were negative for COVID-19, which failed to support the vertical intrauterine transmission. ${ }^{17}$

Existing methods for COVID-19 detection may give false negative results, when the viral load is not high enough, among asymptomatic pregnant women in early stages. Since there is insufficient data on maternal viral load, it is difficult to find out whether the fetus has been infected by maternal viremia in utero before the mother had any clinical manifestation. ${ }^{17}$ Alzamora et al identified COVID-19 in nasopharyngeal swab (16 and 48 hours sample by real-time reverse transcription [RT-PCR]) of neonate and confirmed COVID-19 positivity, but the antibody levels were not elevated up to 5 days of life, which might be due to impaired antibodies and cytokine production in the neonatal period, especially seen in preterm neonates. ${ }^{18}$

Specific IgM antibodies in blood with negative nasopharyngeal swab test (by RT-PCR) have been reported in newborn babies immediately after birth from COVID-19 infected mothers. Since IgM-due to its pentameric structure-does not usually cross the placental barrier, this response indicates transmission of novel coronavirus to the fetus from mother. However, false positive testing may also occur due to alterations in placenta permitting the transmission of IgM. ${ }^{13,19}$ Chen et al reported that COVID-19 nucleic acid titers in placentas of COVID-19 infected mother were undetectable. ${ }^{20}$ Transcriptomic study by Li et al reported extensive repression of COVID-19 receptors (ACE-2) on maternal-fetal interface and fetal organs like lung, liver, and heart. ${ }^{21}$ Wrapp et al reported that the novel 2019-nCoVS binds with higher affinity than SARS-CoVS, which suggests that COVID-19 is more likely to attack placenta and increase the chances of miscarriage. ${ }^{22}$ However, it remains unclear and further studies are required to confirm this. Celik et al reported that absence of caveolin expression in syncytiotrophoblasts prevents the vertical transmission of COVID-19. Caveolae are " $\Omega$ " shaped structure present on cell membrane. It contains caveolin-1 protein that acts as a binding site for COVID-19. Usually, this protein expressed on the alveolo-capillary barrier, but not on the syncytio-capillary barrier. ${ }^{23}$

The most likely way of COVID-19 transmission from mother to infant is likely through respiratory droplets. Hence, during the time of delivery, immediate cord clamping, isolation of neonates, and avoiding skin-to-skin contact with mother can reduce the neonatal infection. ${ }^{24}$ COVID-19 may exist for a longer duration in children's gastrointestinal tract compared with the respiratory system. Continual shedding of COVID-19 in stools of infected children suggests that the virus might be transmitted through contaminated fomites. ${ }^{25}$

Since the virus has not been isolated from breast milk, breastfeeding can be done with social distancing or expressed breast milk feeding to the baby by another care taker using cup and spoon.

\section{Immunological Mechanisms}

Successful pregnancy depends on well-tuned immune adaptations, instead of constant immune suppression. Maternal im- mune system adapted to changes according to the fetal growth and development across different gestational ages such as proinflammatory (embryo implantation and placentation), antiinflammatory (fetal growth), and second pro-inflammatory responses (adapting initiation and parturition) $t$ first, second, and third trimester, respectively. ${ }^{26}$ Cytokine storm is a well-defined feature associated with the COVID-19 infection, which is characterized by elevated plasma concentrations of interleukin (IL)-10, IL-7, IL-2, tumor necrosis factor- $\alpha$, interferon $\gamma$-inducible protein 10 , macrophage inflammatory protein $1 \alpha$, granulocyte-colony stimulating factor, and monocyte chemo attractant protein 1 . Usually, pregnant women during first and third trimester are at pro-inflammatory state; ${ }^{27}$ hence, the cytokine storm induced by COVID-19 may cause harsh inflammatory response in women, which causes postnatal neuronal dysfunctions. ${ }^{28}$

\section{Clinical Manifestations}

In children, high index of suspicion is required to diagnose COVID-19, as the clinical manifestations are similar to other common viral infections. Generally, $95 \%$ of the children show mild or no symptoms. The commonly reported symptoms are fever, sore throat, cough, nasal congestion, and discharge from nose. Respiratory symptoms are least common while gastrointestinal symptoms are more common in children compared with adults. ${ }^{29}$ Febrile seizures have been reported rarely. ${ }^{30}$ Tachypnea is the most common sign of lower respiratory tract involvement in newborns. The severity of illness is usually mild in children, but rarely they may present as sepsis and encephalitis. Compared with older children, severe illness is more common in neonates with crying, irritability, silent hypoxia, neurological symptoms, and feeding difficulties. ${ }^{30-33}$ Concurrent infections with Epstein-Barr virus, rhinoviruses, enterovirus, respiratory syncytial virus, streptococcus pneumonia, and non-SARS coronaviruses are more common in children. ${ }^{34-36}$ Pediatric patients are different from adults in that they often have elevated procalcitonin levels and consolidation in lung with surrounding halo sign on imaging. It indicates that coinfection is more prevalent in pediatric patients. ${ }^{34}$ Perinatal novel coronavirus infection may have severe impact on neonates, such as respiratory distress, premature labor, thrombocytopenia, fetal distress, abnormal liver function, and occasionally death. ${ }^{37}$

\section{Other Unusual Features}

\section{Cytokine Release Syndrome}

It can cause multiple organ dysfunction syndrome leading to death with elevated pro-inflammatory cytokines and inflammatory markers. This complication has rarely been reported in children. ${ }^{38}$

\section{Multisystem Inflammatory Syndrome in Children}

A cluster of adolescents and children who developed a prominent inflammatory response systemically with occasional deaths have been reported in some places of United States and Europe. ${ }^{39}$ This inflammatory response shares 
common features with bacterial meningitis, macrophage activation syndromes, Kawasaki disease, and toxic shock syndrome. MIS-C appears to be a postinfectious cytokine storm with prolonged fever ( 5 days or more), mucocutaneous inflammation signs, hypotension secondary to myocardial dysfunction, evidence of coagulopathy, acute gastrointestinal problems, and elevated inflammatory markers such as erythrocyte sedimentation rate, C-reactive protein, and procalcitonin. However, often there is no evidence of bacterial infection. These patients often had an evidence of novel coronavirus in the form of positive RT-PCR, antigen, or antibody test positivity. ${ }^{40-44}$ The clinical and biochemical aspects of MIS-C are different from Kawasaki disease. ${ }^{45}$ Belhadjer et al reported 35 children affected with MIS-C syndrome who had features of fever and acute heart failure, $28 \%$ of them had comorbidities with prominent gastrointestinal symptoms with release of markers as a result of cytokine storm and macrophage activation. ${ }^{46}$ However, it is unclear whether these manifestations are part of Kawasaki disease with COVID-19 as the stimulating agent or a different syndrome.

\section{Rhabdomyolysis}

It is the leakage of muscle content into extracellular fluid because of skeletal muscle breakdown. Gefen et al observed elevated creatine kinase levels in COVID-19, associated with rhabdomyolysis in a pediatric case. ${ }^{47}$

\section{Pregnancy-Related Complications}

The detrimental effects on the neonates including thrombocytopenia, fetal distress, abnormal liver functions, premature labor, and respiratory distress have been reported. But it is uncertain whether these adverse effects are caused by maternal COVID-19 infection or by direct infection of neonate. Maternal mortality has been documented along with oligohydramnios, perinatal death, preterm birth, miscarriage (including a case in the second trimester), intrauterine growth restriction, ectopic pregnancy, and neonatal death. ${ }^{13-15,37,48-54}$

\section{Imaging Findings}

The radiographic and CT findings play an important role in the COVID-19 diagnosis. Wang et al observed that some children with no clinical symptoms had nonspecific positive chest computed tomography (CT) findings. Other identified findings include interstitial abnormalities, local and bilateral patchy shadowing, ground glass opacities (GGO), and consolidation. ${ }^{55,56} \mathrm{Li}$ et al observed that chest $\mathrm{CT}$ imaging features of COVID-19 infection in preschool children were different from those in adult patients as they had smaller pulmonary consolidations, no ground glass opacities (GGO), and presence of bronchitis/bronchial pneumonia-like changes. Additionally, small airway lesions with uneven lucency of the regional lung lobe or multiple small cystic lucent shadows in the bilateral lower lungs were observed. Chest CT manifestations in preschool children were mostly mild with better outcome after treatment. ${ }^{57}$ Normal findings on chest CT were observed in pediatric patients with mild COVID-19 illness; however, few of them had disease progression on CT scan showing more localized GGO extent, lower GGO attenuation, and relatively rare interlobular septal thickening. ${ }^{5}$ Feng et al reported smaller nodular GGO in COVID-19 pediatric patients. However, other features such as interlobular septa thickening, consolidation, GGO with consolidation were also observed. ${ }^{58}$ Other chest imaging findings reported in these pediatric patients include interstitial changes, diffuse consolidation (white lung), bronchopneumonia with mycoplasma infection and Pleural effusion. Lung lesions completely resolve with recovery in most patients. However, to protect the children from radiation, the usage of chest CT in pediatric patients with COVID-19 should be more cautious. ${ }^{5}$

\section{Treatment Modalities}

As the vaccine is still being developed against SARS-n-CoV-2 and with the lack of a definitive therapy specifically targeting this virus, the treatment is mainly symptomatic and supportive. Oxygen therapy is the most efficient treatment in critically ill neonates and children. Early treatment reduces the complications like acute respiratory distress syndrome and multiorgan dysfunction. There is no clear-cut evidence of definite improvement with antiviral therapy in pediatric COVID-19 cases till date. ${ }^{59}$ Remdesivir, favipiravir, and ribavirin are the nucleotide analogs effective against premature termination of COVID-19 RNA transcriptome. Jean et al reported that remdesivir (200 mg/day 1 and $100 \mathrm{mg} /$ day 2 onward) was used to treat a COVID-19 patient with progressive pulmonary manifestations on the 7th day of hospitalization with clinical improvement. Side-effects such as nausea, vomiting, including severe ones like rectal hemorrhage, and hepatic toxicity have been reported recently. Favipiravir (1,600 mg/twice/1 day, $600 \mathrm{mg} / \mathrm{twice} / 2-5$ days, and $600 \mathrm{mg} /$ once on the 6th day) was used for the treatment of COVID-19 in China. Ribavirin is also an antiviral drug, but it may cause anemia and harmful in patients with respiratory distress. ${ }^{60}$

Corticosteroids were used to treat patients with COVID-19 infection in a few reports. Its possible benefit is by reducing the inflammatory induced lung injury; ${ }^{27}$ however, few studies suggested that treating with corticosteroids may not have any effect on mortality rather it may delay the viral clearance in SARS and middle east respiratory syndrome patients. ${ }^{61-63}$ WHO interim guidelines do not suggest corticosteroids therapy except in severe cases. ${ }^{64}$ Intravenous immunoglobulin (IVIG) is being tried to treat the neonates and severely ill children. ${ }^{65}$ Recent study reported that Bruton's tyrosine kinase (BTK) controls the macrophage activation leading to elevated levels of IL-6 in COVID-19 adults; hence, acalabrutinib (selective BTK inhibitor) may be used as a treatment option in severe SARS-n-COV-2 infection. ${ }^{66}$ However, its use has not shown benefit in neonatal and pediatric cases. Few studies reported that COVID-19 host cell entry through spike proteins have offered insight for vaccine development and usage of various protease inhibitors. ${ }^{67}$ Either lopinavir (400 mg/twice/ 14 days) or ritonavir ( $100 \mathrm{mg} /$ twice/14 days) alone may not be beneficial for COVID-19 treatment; however, the regimen of 
lopinavir and ritonavir together may be effective. Alternatively, hydroxychloroquine (200 $\mathrm{mg} /$ thrice/daily) plus azithromycin ( $500 \mathrm{mg} / 1$ day, followed by $250 \mathrm{mg}$ once daily on $2-5$ days) has emerged as an alternate treatment option with excellent clinical efficacy in Chinese patients with COVID-19 infection. ${ }^{60}$ Several studies reported the usage of convalescent plasma (CP) therapy for SARS-n-COV-2 treatment. It improved oxygenation and lymphocyte count and reduced inflammatory markers/viral load. ${ }^{68}$ Duan et al described improvement in several parameters compared with pretransfusion including increased lymphocyte counts $(0.65 \times 109 / \mathrm{L}$ vs. $0.76 \times 109 / \mathrm{L})$ and decreased C-reactive protein ( 55.98 vs. $18.13 \mathrm{mg} / \mathrm{L}$ ). There was improvement in the lung lesions within 7 days on radiological examinations. Additionally, undetectable viral load in patients with viremia and no complications were observed. ${ }^{69}$ The mechanism of action is not fully understood. However, anti-SARS-IgM and IgG directly neutralizes the virus. ${ }^{70}$ However, it is important to formulate individual therapy regimens. $\mathrm{CP}$ can only be used empirically by strict screening for indications monitoring the transfusion process and with strict recording of outcome. ${ }^{71}$ The mandatory conditions for donation of CP are that the donor must have been diagnosed with COVID-19 infection and recovered at least 14 days earlier, male and nulliparous female patients with negative history of blood transfusion. Careful evaluation of the donor is recommended. The antibody titer should be at least 1:320, with negative results of biological qualification tests, hepatitis $\mathrm{A}, \mathrm{B}$, parvo virus, and proper maintenance of $\mathrm{CP}$ units. ${ }^{72}$ But most of these studies were described in adults. It is uncertain whether the CP therapy will be effective in critically ill pediatric patients.

\section{Prognostic Factors}

Laboratory abnormalities were not widely reported in COVID-19 infected children compared with adult COVID19 patients. The burden of the disease severity seems to be low in children compared with adults. Dong et al reported that $10 \%$ of the neonates who tested positive for SARS-nCOV2 probably are at high risk requiring ventilator support and other interventions. ${ }^{6}$ Children with adverse SARS-nCOV-2 clinical course show elevated procalcitonin, CRP, and LDH levels similar to adult patients with COVID-19. ${ }^{73}$ Additionally, positive D-dimer, elevated prothrombin time were observed in COVID-19 infected children. ${ }^{74}$ IL-6, IL-10, and serum ferritin levels may help to prognosticate among infected children. But none of the studies have reported elevated serum ferritin levels in neonates. ${ }^{75} \mathrm{Li}$ et al reported that the children with SARS-n-cov-2 pneumonia had elevated levels of CD8 + T cells compared with those with respiratory syncytial virus pneumonia. This activation plays a crucial role in the development of symptoms among children with SARS-n-COV-2 pneumonia; hence, it could be used as a biomarker to predict the prognosis of COVID-19 infection. ${ }^{76}$

\section{Conclusion}

COVID-19 affects people of all age groups. Most of the neonatal and pediatric cases are seen with mild symptoms as clusters in family, usually have rapid recovery and good prognosis. Early isolation of neonate from infected mother should be done since there no definite evidence of intrauterine vertical transmission. Breast milk feeding can be given since there is no evidence of virus in breast milk. Moreover, vaccine development and identification of effective antiviral drugs against COVID-19 are important for improving the outcome.

\section{Funding \\ None.}

Conflict of Interest

None declared.

\section{References}

1 Lipsitch M, Swerdlow DL, Finelli L. Defining the epidemiology of COVID-19 - studies needed. N Engl J Med 2020;382(13): 1194-1196

2 Wong SH, Lui RN, Sung JJ. COVID-19 and the digestive system. J Gastroenterol Hepatol 2020;35(05):744-748

3 Wang T, Du Z, Zhu F, et al. Comorbidities and multi-organ injuries in the treatment of COVID-19. Lancet 2020;395(10228):e52

4 Cascella M, Rajnik M, Cuomo A, Dulebohn SC, Di Napoli R. Features, Evaluation and Treatment Coronavirus (COVID-19). Treasure Island, FL: Statpearls Publishing; 2020

5 Duan YN, Zhu YQ, Tang LL, Qin J. CT features of novel coronavirus pneumonia (COVID-19) in children. Eur Radiol 2020;30(08): 4427-4433

6 Dong Y, Mo X, Hu Y, et al. Epidemiology of COVID-19 among children in China. Pediatrics 2020;145(06):e20200702

7 The Novel Coronavirus Pneumonia Emergency Response Epidemiology Team. The epidemiological characteristics of an outbreak of 2019 novel coronavirus diseases (COVID-19) -China, 2020. China CDC Weekly 2020;2(08):113-122

8 Guan WJ, Ni ZY, Hu YChina Medical Treatment Expert Group for Covid-19, et al. Clinical characteristics of coronavirus disease 2019 in China. N Engl J Med 2020;382(18):1708-1720

9 Zimmermann P, Curtis N. Coronavirus infections in children including COVID-19: an overview of the epidemiology, clinical features, diagnosis, treatment and prevention options in children. Pediatr Infect Dis J 2020;39(05):355-368

10 Wan Y, Shang J, Graham R, Baric RS, Li F. Receptor recognition by the novel coronavirus from Wuhan: an analysis based on decadelong structural studies of SARS coronavirus. J Virol 2020;94(07): e00127-e20

11 Mason RJ. Pathogenesis of COVID-19 from a cell biology perspective. Eur Respir J 2020;55(04):2000607

12 Li G, He X, Zhang L, et al. Assessing ACE2 expression patterns in lung tissues in the pathogenesis of COVID-19. J Autoimmun 2020; 112:102463

13 Dashraath P, Wong JLJ, Lim MXK, et al. Coronavirus disease 2019 (COVID-19) pandemic and pregnancy. Am J Obstet Gynecol 2020; 222(06):521-531

14 Chen H, Guo J, Wang C, et al. Clinical characteristics and intrauterine vertical transmission potential of COVID-19 infection in nine pregnant women: a retrospective review of medical records. Lancet 2020;395(10226):809-815

15 Schwartz DA. An analysis of 38 pregnant women with COVID-19, their newborn infants, and maternal-fetal transmission of SARSCoV-2: maternal coronavirus infections and pregnancy outcomes. Arch Pathol Lab Med 2020;144(07):799-805

16 Zhou P, Yang XL, Wang XG, et al. A pneumonia outbreak associated with a new coronavirus of probable bat origin. Nature 2020;579 (7798):270-273 
17 Wang S, Guo L, Chen L, et al. A case report of neonatal 2019 coronavirus disease in China. Clin Infect Dis 2020;71(15): 853-857

18 Alzamora MC, Paredes T, Caceres D, Webb CM, Valdez LM, La Rosa M. Severe COVID-19 during pregnancy and possible vertical transmission. Am J Perinatol 2020;37(08):861-865

19 Zeng H, Xu C, Fan J, et al. Antibodies in infants born to mothers with COVID-19 pneumonia. JAMA 2020;323(18):1848-1849

20 Chen S, Huang B, Luo DJ, et al. [Pregnancy with new coronavirus infection: clinical characteristics and placental pathological analysis of three cases]. Zhonghua Bing Li Xue Za Zhi 2020;49(05): 418-423

21 Li M, Chen L, Zhang J, Xiong C, Li X. The SARS-CoV-2 receptor ACE2 expression of maternal-fetal interface and fetal organs by singlecell transcriptome study. PLoS One 2020;15(04):e0230295

22 Wrapp D, Wang N, Corbett KS, et al. Cryo-EM structure of the 2019-nCoV spike in the prefusion conformation. Science 2020; 367(6483):1260-1263

23 Celik O, Saglam A, Baysal B, et al. Factors preventing materno-fetal transmission of SARS-CoV-2. Placenta 2020;97:1-5

24 Chandrasekharan P, Vento M, Trevisanuto D, et al. Neonatal resuscitation and postresuscitation care of infants born to mothers with suspected or confirmed SARS-CoV-2 Infection. Am J Perinatol 2020;37(08):813-824

25 Xing YH, Ni W, Wu Q, et al. Prolonged viral shedding in feces of pediatric patients with coronavirus disease 2019. J Microbiol Immunol Infect 2020;53(03):473-480

26 Mor G, Aldo P, Alvero AB. The unique immunological and microbial aspects of pregnancy. Nat Rev Immunol 2017;17(08):469-482

27 Huang C, Wang Y, Li X, et al. Clinical features of patients infected with 2019 novel coronavirus in Wuhan, China. Lancet 2020;395 (10223):497-506

28 Liu H, Wang LL, Zhao SJ, Kwak-Kim J, Mor G, Liao AH. Why are pregnant women susceptible to COVID-19? An immunological viewpoint. J Reprod Immunol 2020;139:103122

29 Liguoro I, Pilotto C, Bonanni M, et al. SARS-COV-2 infection in children and newborns: a systematic review. Eur J Pediatr 2020; 179(07):1029-1046

30 Garazzino S, Montagnani C, Donà DItalian SITIP-SIP Pediatric Infection Study Group Italian SITIP-SIP SARS-CoV-2 Paediatric Infection Study Group*, et al. Multicentre Italian study of SARS$\mathrm{CoV}-2$ infection in children and adolescents, preliminary data as at 10 April 2020. Euro Surveill 2020;25(18):2000600

31 Lorenz N, Treptow A, Schmidt S, et al. Neonatal early-onset infection with SARS-CoV-2 in a newborn presenting with encephalitic symptoms. Pediatr Infect Dis J 2020;39(08):e212

32 Chacón-Aguilar R, Osorio-Cámara JM, Sanjurjo-Jimenez I, González-González C, López-Carnero J, Pérez-Moneo-Agapito B. COVID19: fever syndrome and neurological symptoms in a neonate. An Pediatr (Engl Ed) 2020; Jun; 92(06):373-374

33 Sinelli M, Paterlini G, Citterio M, Di Marco A, Fedeli T, Ventura ML. Early neonatal SARS-CoV-2 infection manifesting with hypoxemia requiring respiratory support. Pediatrics 2020;146(01):e20201121

34 Xia W, Shao J, Guo Y, Peng X, Li Z, Hu D. Clinical and CT features in pediatric patients with COVID-19 infection: different points from adults. Pediatr Pulmonol 2020;55(05):1169-1174

35 DeBiasi RL, Song X, Delaney M, et al. Severe COVID-19 in children and young adults in the Washington, DC Metropolitan Region. J Pediatr 2020;223:199-203.e1

36 Garazzino S, Montagnani C, Donà DItalian SITIP-SIP Pediatric Infection Study Group Italian SITIP-SIP SARS-CoV-2 Paediatric Infection Study Group*, et al. Multicentre Italian study of SARSCoV-2 infection in children and adolescents, preliminary data as at 10 April 2020. Euro Surveill 2020;25(18):2000600

37 Zhu H, Wang L, Fang C, et al. Clinical analysis of 10 neonates born to mothers with 2019-nCoV pneumonia. Transl Pediatr 2020;9 (01):51-60
38 Ye Q, Wang B, Mao J. The pathogenesis and treatment of the 'Cytokine Storm' in COVID-19. J Infect 2020;80(06):607-613

39 Pain CE, Felsenstein S, Cleary G, et al. Novel paediatric presentation of COVID-19 with ARDS and cytokine storm syndrome without respiratory symptoms. Lancet Rheumatol 2020;2(07):e376-e379

40 New York State Governor Cuomo announces state is helping to develop the national criteria for identifying and responding to COVID-related illness in children. Available at: https://www.governor.ny.gov/news/governor-cuomo-announces-state-helpingdevelop-national-criteria-identifying-and-responding. Accessed September 1, 2020

41 Paediatric Intensive Care Society PICS statement: increased number of reported cases of novel presentation of multisystem inflammatory disease. Available at: https://pccsociety.uk/news/ pics-statement-regarding-novel-presentation-of-multi-systeminflammatory-disease/. Accessed April 27, 2020

42 Mahase E. COVID-19: concerns grow over inflammatory syndrome emerging in children. BMJ 2020;369:m1710

43 Daskalakis DC2020 health alert \#13: pediatric multi-system inflammatory syndrome potentially associated with COVID-19 Pediatric Intensive Care Society. PICS Statement: increased number of reported cases of novel presentation of multi-system inflammatory disease Available at: https://picsociety.uk/wpcontent/uploads/2020/04/PICS-statement-re-novel-KDC19-presentation-v2-27042020.pdf. Accessed April 27, 2020

44 Riphagen S, Gomez X, Gonzalez-Martinez C, Wilkinson N, Theocharis P. Hyperinflammatory shock in children during COVID-19 pandemic. Lancet 2020;395(10237):1607-1608

45 Verdoni L, Mazza A, Gervasoni A, et al. An outbreak of severe Kawasaki-like disease at the Italian epicentre of the SARS-CoV-2 epidemic: an observational cohort study. Lancet 2020;395 (10239):1771-1778

46 Belhadjer Z, Méot M, Bajolle F, et al. Acute heart failure in multisystem inflammatory syndrome in children (MIS-C) in the context of global SARS-CoV-2 pandemic. Circulation 2020;142:429-436

47 Gefen AM, Palumbo N, Nathan SK, Singer PS, Castellanos-Reyes LJ, Sethna CB. Pediatric COVID-19-associated rhabdomyolysis: a case report. Pediatr Nephrol 2020;35(08):1517-1520

48 Liu D, Li L, Wu X, et al. Pregnancy and perinatal outcomes of women with coronavirus disease (COVID-19) pneumonia: a preliminary analysis. AJR Am J Roentgenol 2020;215(01):127-132

49 Di Mascio D, Khalil A, Saccone G, et al. Outcome of coronavirus spectrum infections (SARS, MERS, COVID-19) during pregnancy: a systematic review and meta-analysis. Am J Obstet Gynecol MFM 2020;2(02):100107

50 Chen L, Li Q Zheng D, et al. Clinical characteristics of pregnant women with COVID-19 in Wuhan, China. N Engl J Med 2020;382(25):e100

51 Baud D, Greub G, Favre G, et al. Second-trimester miscarriage in a pregnant woman with SARS-CoV-2 infection. JAMA 2020;323 (21):2198-2200

52 Hantoushzadeh S, Shamshirsaz AA, Aleyasin A, et al. Maternal death due to COVID-19. Am J Obstet Gynecol 2020;223(01):109. e1-109.e16

53 Li J, Wang Y, Zeng Y, et al. Critically ill pregnant patient with COVID-19 and neonatal death within two hours of birth. Int J Gynaecol Obstet 2020;150(01):126-128

54 Aliji N, Aliu F. Oligohydramnion in COVID19. Eur J Obstet Gynecol Reprod Biol 2020;249:102

55 Lu X, Zhang L, Du H. Chinese Pediatric Novel Coronavirus Study Team. et al.. SARS-CoV-2 Infection in Children. N Engl J Med 2020; 382(17):1663-1665

56 Bernheim A, Mei X, Huang M, et al. Chest CT findings in coronavirus disease-19 (COVID-19): relationship to duration of infection. Radiology 2020;295(03):200463

57 Li Y, Cao J, Zhang X, Liu G, Wu X, Wu B. Chest CT imaging characteristics of COVID-19 pneumonia in preschool children: a retrospective study. BMC Pediatr 2020;20(01):227 
58 Feng K, Yun YX, Wang XF, et al. [Analysis of CT features of 15 Children with 2019 novel coronavirus infection]. Zhonghua Er Ke Za Zhi 2020;58(00):E007

59 Yu Y, Chen P. Coronavirus disease 2019 (COVID-19) in neonates and children from China: a review. Front Pediatr 2020;8:287

60 Jean SS, Lee PI, Hsueh PR. Treatment options for COVID-19: the reality and challenges. J Microbiol Immunol Infect 2020;53(03): 436-443

61 Stockman LJ, Bellamy R, Garner P. SARS: systematic review of treatment effects. PLoS Med 2006;3(09):e343

62 Lansbury LE, Rodrigo C, Leonardi-Bee J, Nguyen-Van-Tam J, Shen Lim W. Corticosteroids as adjunctive therapy in the treatment of influenza: an updated cochrane systematic review and metaanalysis. Crit Care Med 2020;48(02):e98-e106

63 Arabi YM, Mandourah Y, Al-Hameed FSaudi Critical Care Trial Group, et al. Corticosteroid therapy for critically ill patients with middle east respiratory syndrome. Am J Respir Crit Care Med 2018;197(06):757-767

64 World Health Organization Clinical management of severe acute respiratory infection when novel coronavirus (2019-nCoV) infection is suspected: interim guidance, 28 January 2020 . Available at: https://www.who.int/docs/default-source/coronaviruse/clinicalmanagement-of-novel-cov.pdf. Accessed March 13, 2020

65 Zhang J, Yang Y, Yang N, et al. Effectiveness of intravenous immunoglobulin for children with severe COVID-19: a rapid review. Ann Transl Med 2020;8(10):625

66 Roschewski M, Lionakis MS, Sharman JP, et al. Inhibition of Bruton tyrosine kinase in patients with severe COVID-19. Sci Immunol 2020;5(48):110

67 Dhama K, Sharun K, Tiwari R, et al. COVID-19, an emerging coronavirus infection: advances and prospects in designing and developing vaccines, immunotherapeutics, and therapeutics. Hum Vaccin Immunother 2020;16(06):1232-1238

68 Ahn JY, Sohn Y, Lee SH, et al. Use of convalescent plasma therapy in two COVID-19 patients with acute respiratory distress syndrome in Korea. J Korean Med Sci 2020;35(14):e149

69 Duan K, Liu B, Li C, et al. Effectiveness of convalescent plasma therapy in severe COVID-19 patients. Proc Natl Acad Sci USA 2020;117(17):9490-9496

70 Yuen KS, Ye ZW, Fung SY, Chan CP, Jin DY. SARS-CoV-2 and COVID19: the most important research questions. Cell Biosci 2020;10:40

71 Li XY, Du B, Wang YS, et al. The keypoints in treatment of the critical coronavirus disease 2019 patient(2). Zhonghua Jie He He Hu Xi Za Zhi 2020;43(04):277-281

72 Franchini M, Marano G, Velati C, Pati I, Pupella S, Maria Liumbruno G. Operational protocol for donation of anti-COVID-19 convalescent plasma in Italy. Vox Sang 2020. Doi: 10.1111/vox.12940

73 Henry BM, de Oliveira MHS, Benoit S, Plebani M, Lippi G. Hematologic, biochemical and immune biomarker abnormalities associated with severe illness and mortality in coronavirus disease 2019 (COVID-19): a meta-analysis. Clin Chem Lab Med 2020;58(07):1021-1028

74 Henry BM, Vikse J, Benoit S, Favaloro EJ, Lippi G. Hyperinflammation and derangement of renin-angiotensin-aldosterone system in COVID-19: a novel hypothesis for clinically suspected hypercoagulopathy and microvascular immunothrombosis. Clin Chim Acta 2020;507:167-173

75 Henry BM, Benoit SW, de Oliveira MHS, et al. Laboratory abnormalities in children with mild and severe coronavirus disease 2019 (COVID-19): a pooled analysis and review. Clin Biochem 2020;81:1-8

$76 \mathrm{Li} \mathrm{H}$, Chen K, Liu M, Xu H, Xu Q. The profile of peripheral blood lymphocyte subsets and serum cytokines in children with 2019 novel coronavirus pneumonia. J Infect 2020;81(01):115-120 